\title{
Metastable states and activated dynamics in thin-film adhesion to patterned surfaces
}

\author{
Stefan B Lindström, Lars Johansson and Nils R. Karlsson
}

\section{Linköping University Post Print}

\section{Tweet}

N.B.: When citing this work, cite the original article.

Original Publication:

Stefan B Lindström, Lars Johansson and Nils R. Karlsson, Metastable states and activated dynamics in thin-film adhesion to patterned surfaces, 2014, Physical Review E. Statistical, Nonlinear, and Soft Matter Physics, (89), 062401-1-062401-11.

http://dx.doi.org/10.1103/PhysRevE.89.062401

Copyright: American Physical Society

http://www.aps.org/

Postprint available at: Linköping University Electronic Press

http://urn.kb.se/resolve?urn=urn:nbn:se:liu:diva-110756 


\title{
Metastable states and activated dynamics in thin-film adhesion to patterned surfaces
}

\author{
Stefan B. Lindström, " Lars Johansson, and Nils R. Karlsson \\ Mechanics, Department of Management and Engineering, the Institute of Technology, Linköping University, SE-581 83 Linköping, Sweden
}

(Received 12 February 2014; published 6 June 2014)

\begin{abstract}
We consider adhesion due to London-van der Waals attraction between a thin film and a patterned surface with nanometer asperities. Depending on the surface topography and the stiffness of the film, three regimes of adhesion are identified: complete contact adhesion, partial contact adhesion, and glassy adhesion. For complete contact adhesion, the film conforms to the undulations of the surface, whereas for partial contact and glassy adhesion, the adhesive interface breaks down into microscopic areas of contact. When a film in the glassy regime is peeled off the surface, metastable states develop at which the crack front becomes arrested, analogously to the frustrated motion of the three-phase contact line across a heterogeneous surface. For this glassy regime, we use transition state theory to model the thermally activated progression of the crack front. This theoretical treatment suggests that the rate of the adhesive failure increases exponentially with the applied force.
\end{abstract}

DOI: 10.1103/PhysRevE.89.062401

PACS number(s): 62.20.F-, 68.35.Np, 82.20.Db, 68.60.Bs

\section{INTRODUCTION}

From Scotch ${ }^{\mathrm{TM}}$ tape and cling wrap to the footpads that allow spiders and geckos to climb vertical walls [1], a dry adhesive serves as a removable joint between dissimilar materials. These adhesives deform against a surface and form weak physical bonds with it. Their detachment is governed by the collaborative failure of these bonds, which are localized to microscopic areas of contact due to the roughness of the interface between any two materials. The dry adhesion of thin films against patterned surfaces can be regarded as an idealization of rough interface adhesion, but is also of great interest and importance when considering interactions between sheetlike macromolecules and functionalized, micropatterned surfaces, including the application of substrate-regulated morphology of graphene multilayers [2].

Similarly to the brittle fracture of solids [3] and the yielding of soft materials [4,5], adhesive failure is controlled by a succession of microscopic events which trigger macroscopic failure. As inspired by contact mechanics [6] and fracture mechanics [3,7], the conventional view is that adhesive failure is initiated when the energy dissipated during fracture per unit of newly created fracture surface area exceeds some critical value. Such an equilibrium description of adhesive stability cannot be reconciled with the logarithmic force-rate dependence that is typically observed for adhesive failure of soft interfaces [8,9], which is consistent with Arrhenius-type behavior for the dissociation of microcontacts. This rate dependence is captured by kinetic models, which describe adhesive failure as a sequence of bond-breaking events [10,11]. Still, a quantitative model taking into account the nature of the physical bonds, the surface topography and the finite flexibility of the materials has yet to emerge.

In this work we formulate a model for adhesion and adhesive failure dynamics of a flexible thin film adhering through London-van der Waals (vdW) attraction to a surface with nanoscale asperities. The underlying surface is modeled as a half space, and two types of micropatterns are considered: a periodic grid of smooth protrusions and a raster of parallel

\footnotetext{
*stefan.lindstroem@gmail.com
}

ridges. For the adhesive failure, we consider peel-off by an external force applied to one of the film's edges. The variation of the potential of the adhesive system due to the surface undulations is taken into account. The colloidal length scale of the system under considerations implies that thermal fluctuations must be considered; these may activate state transitions [12] and thus affect adhesive stability [13]. This is analogous to the effect of thermal fluctuations on the stability of intermolecular bonds [14] and bonds between weakly attracting colloidal particles [4,5].

The peel-off of a thin film by means of a force applied to its edge was described by Kendall [15] as an energy balance, including the interaction energy and the elastic energy of a film contacting a flat surface. Inertia, viscoelasticity of the film, and surface effects from the microscale topography or chemical heterogeneity were neglected. Building on the conceptual view of Kendall, the development of models for nanothick adhesive films has to a large extent been driven by the study of adhesive systems found in nature, and the gecko's adhesive footpads in particular. The footpad of the gecko is covered by hairlike setae which branch into thinner filaments. Each seta subdivides into 300 to 1000 filaments at the finest level of organization [16]. The thinnest filaments terminate in a flat, triangularly shaped contacting element [8,17-19] called a spatula. These spatulae were modeled as thin films in previous work [20,21]. The strain energy of the film modifies the total energy of the gecko's adhesion system, yielding a smaller effective interaction energy than that expected for a flat surface [20]. By introducing a Lennard-Jones (12-6) potential for interaction of material points, a quantitative model of the governing principles at the microscale was developed [21].

The present work focuses on the generic aspects of dry adhesion between a thin film and a patterned surface. We investigate the vdW interaction energy for the limiting cases of a very flexible and a very stiff film, respectively (Sec. II). This result is used for computing the potential of the thin film while being peeled off from the patterned surface by a force applied to one edge of the film (Sec. II). This surface potential fluctuates due to localized adhesion interactions, so that a landscape of potential maxima and minima is formed. We quantify these fluctuations and employ transition state theory [12] to model the activated dynamics of the crack 
front of the adhesive interface during peel-off (Sec. III). The theory developed for the adhesive failure is applied to the contact elements of the gecko adhering to a micropatterned glass surface (Sec. IV B).

\section{THIN-FILM ADHESION}

In this section we study the interaction potential of a flexible thin film against a patterned surface due to vdW attraction. It is assumed that the process of adhesion is such that the free energy is minimized. This is realistic for nanoscale, thermal systems. The discussion below is also valid for macroscopic adhesion when a film and surface is brought into contact in such a way that the interaction energy is allowed to be minimized.

\section{A. Van der Waals interactions}

Consider two continuous bodies of electrostatically neutral condensed matter, occupying the spatial regions $\Omega$ and $\Omega^{\prime}$, respectively. We are concerned with the interaction forces between the bodies, which are modeled in terms of an interaction potential $V$, consisting of a $\mathrm{vdW}$ attraction and a repulsive part $\phi_{\mathrm{P}}$ [22]. The interaction potential between two infinitesimal volume elements, $d x d y d z$ and $d x^{\prime} d y^{\prime} d z^{\prime}$ located at the positions $\vec{x} \in \Omega$ and $\vec{x}^{\prime} \in \Omega^{\prime}$, respectively, is then written as

$$
d V=\left[\phi_{\mathrm{P}}(\rho)-\frac{A}{\pi^{2} \rho^{6}}\right] d x^{\prime} d y^{\prime} d z^{\prime} d x d y d z,
$$

where $\rho=\left|\vec{x}-\vec{x}^{\prime}\right|$ and $A$ is the Hamaker constant. In Eq. (1), the term $-A / \pi^{2} \rho^{6}$ represents the vdW attraction, while $\phi_{\mathrm{P}}(\rho)$ represents Pauli repulsion, which is assumed to approach $\phi_{\mathrm{P}}(\rho)=\phi_{0} \Theta(\delta-\rho)$, where $\Theta$ is Heaviside's step function and $\phi_{0}$ is a large constant. This ensures that when the interaction of two macroscopic bodies is modeled by integrating Eq. (1), they can come no closer than $\delta$ at any point. A condition where the distance between two bodies is $\delta$ in at least one location, but with no contact forces, is called grazing contact. Since the two bodies are prohibited from being closer than a distance $\delta$ at any point, only the vdW term of Eq. (1) contributes to the work involved in moving the bodies relative to each other. For the remainder of this work, only the vdW part of Eq. (1) will be considered.

\section{B. Interaction energy between a film and a half space}

The interaction energy $\gamma$ is introduced as the negative of the integral of the vdW term of Eq. (1) over two interacting bodies. For two half spaces separated by the distance $\delta$, we have

$$
\gamma=\frac{A}{12 \pi \delta^{2}}
$$

with the units of energy per surface area [23, p. 254]. Note that $\delta$ is the equilibrium distance between the bodies if the complete potential according to Eq. (1) is taken into account. Moreover, $\gamma$ is the work per unit area of the vdW forces when the bodies are brought into grazing contact from a large distance. For a film of thickness $d$ at a distance $\delta$ from a half space, we obtain

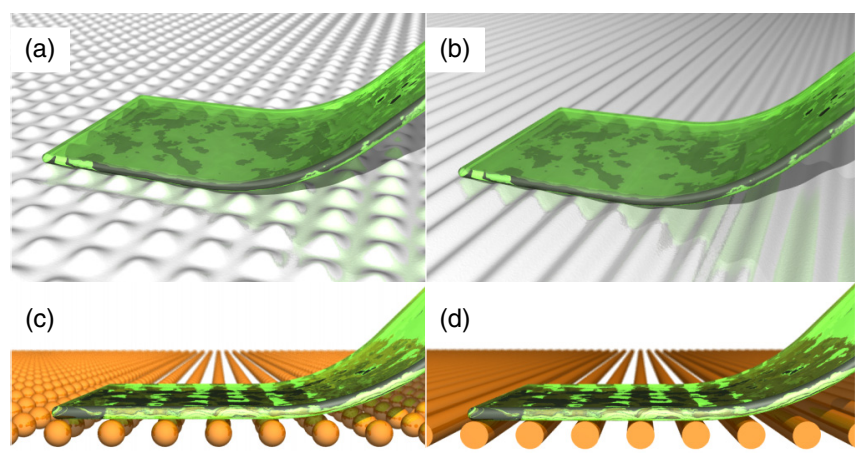

FIG. 1. (Color online) (a) Rendering of a film adhering to a grid of protrusions with aspect ratio $\lambda / b=5$. (b) A film adhering to a corrugated surface with aspect ratio $\lambda / b=5$. (c) Model for surfacefilm interactions using a grid of spheres. (d) Model for surface-film interactions using a raster of cylinders.

by superposition:

$$
\gamma=\frac{A}{12 \pi \delta^{2}}-\frac{A}{12 \pi(d+\delta)^{2}} \approx \frac{A}{12 \pi \delta^{2}},
$$

where the approximation giving the last form is valid for $d \gg \delta$.

\section{Conforming contact against a patterned surface}

In this section we investigate the ability of a thin film to adhere to a macroscopically flat, rigid surface with a topographic micropattern. To this end, we compare the work per unit area $\gamma$ available from vdW attraction as a thin film is brought into contact with a patterned half space to the elastic energy that is stored per unit area of the film when it is bent to conform to the surface undulations. Initially, the surface pattern is restricted to a regular grid of protrusions [Fig. 1(a)]. A corrugated surface [Fig. 1(b)] is considered in Sec. IV A.

As a model surface topography, we use

$$
f(x, y)=b \cos ^{2} \frac{\pi x}{\lambda} \cos ^{2} \frac{\pi y}{\lambda},
$$

which is a square grid of protrusions with height $b$ and separation $\lambda$. Let $S$ denote the nominal adhesion domain in the $x y$ plane, and let $A_{S}=\int_{S} d x d y$ be the nominal contact area.

Considering first the interaction energy, we wish to calculate $\gamma$ for an adhered film that follows the underlying surface $z=f(x, y)$. We assume that the slopes of the protrusions are gentle, i.e., $b \ll \lambda$. Also noting that $\mathrm{vdW}$ interactions have short range, the interaction energy can be calculated using a flat surface against a parallel flat surface assumption locally. The remaining difference to Eq. (3) is then that the actual area of the undulating surface is larger than the nominal area so that [24, p. 153]

$$
\gamma \approx \frac{A}{12 \pi \delta^{2}} \frac{1}{A_{S}} \int_{S} \sqrt{1+\left(\frac{\partial f}{\partial x}\right)^{2}+\left(\frac{\partial f}{\partial y}\right)^{2}} d x d y .
$$

Since $0 \leqslant(\partial f / \partial x)^{2}+(\partial f / \partial y)^{2} \leqslant \pi^{2} b^{2} / \lambda^{2}$, we have

$$
\frac{A}{12 \pi \delta^{2}} \leqslant \gamma \leqslant \frac{A}{12 \pi \delta^{2}} \sqrt{1+\frac{\pi^{2} b^{2}}{\lambda^{2}}} .
$$


Next, the elastic energy $W_{\mathrm{e}}$ of a thin film bent to conform completely to the protrusions is considered. The film is modeled as a Kirchhoff plate with Young's modulus $E_{\mathrm{Y}}$ and Poisson's ratio $v$. The plate stiffness is $D=E_{\mathrm{Y}} d^{3} / 12\left(1-v^{2}\right)$. The strain energy of a plate conforming to a patterned surface generally includes both bending and in-plane strain contributions [2]. Here, however, we consider thermal systems that are allowed to relax into equilibrium, leaving no residual tangential forces between film and surface. Kirchhoff plate theory then infers that there is no in-plane contribution to the strain energy. The elastic bending energy stored in the plate is [25]

$$
W_{\mathrm{e}}=\frac{D}{2} \int_{S}\left[\left(\nabla^{2} f\right)^{2}-2(1-v)\left|H_{f}\right|\right] d x d y,
$$

where $H_{f}$ denotes the Hessian matrix of $f$, so that the Hessian determinant of $f$ is

$$
\left|H_{f}\right|=\frac{\partial^{2} f}{\partial x^{2}} \frac{\partial^{2} f}{\partial y^{2}}-\left(\frac{\partial^{2} f}{\partial x \partial y}\right)^{2} .
$$

An effective interaction energy is defined by [20] $\gamma_{\text {eff }}=\gamma-$ $W_{\mathrm{e}} / A_{S}$, i.e., by reducing the interaction energy with the energy that goes into deformation of the film. The amplitude of the surface undulations and the plate stiffness of the film thus determine the effective interaction energy [21].

For a film adhering to the surface topography of Eq. (4), at the equilibrium distance $\delta$, the middle surface will bend into the same shape $f(x, y)$ as the underlying topography, offset by a distance $\delta+d / 2$. Using Eq. (4) in (7) and integrating over a square region of side length $\lambda$, the elastic energy per unit area for a film deformed to conform to the assumed surface topography is

$$
\frac{W_{\mathrm{e}}}{A_{S}}=\frac{\pi^{4} D b^{2}}{\lambda^{4}} .
$$

We now form a dimensionless number which captures the ability of the film to conform to the model surface. This flexibility ratio is defined as

$$
r_{\mathrm{f}} \equiv \frac{\gamma}{W_{\mathrm{e}} / A_{S}}=\frac{\lambda^{4}}{\lambda_{0}^{4}}, \quad \lambda_{0}=\left(\frac{12 \pi^{5} D b^{2} \delta^{2}}{A}\right)^{1 / 4}
$$

where the interaction energy is estimated by $\gamma=A / 12 \pi \delta^{2}$ to an accuracy quantified by Eq. (6). A flexibility ratio $r_{\mathrm{f}}=1$ represents the limiting case when $\gamma$ is just large enough to maintain a grazing contact from top to bottom of the protrusions. When $r_{\mathrm{f}} \ll 1$ the film is essentially rigid, while for the case $r_{\mathrm{f}} \gg 1, \gamma$ is by a large margin sufficient to bend the film to conform to the surface. A transition from the rigid to the flexible regime is expected at $r_{\mathrm{f}}=1$, for which $\lambda=\lambda_{0}$. This makes clear the physical meaning of the characteristic wavelength $\lambda_{0}$; the film will behave as rigid at protrusion length scales below $\lambda_{0}$. Considering the above discussion, we postulate that the adhesive interface between a film and a rigid surface breaks up into microscopic areas of contact when $r_{\mathrm{f}} \lesssim 1$.

\section{Nonconforming contact against a patterned surface}

Consider a half space with a surface topography given by Eq. (4) and a film aligned with the plane of this surface. Moreover, consider the case $r_{\mathrm{f}} \ll 1$, so that the film behaves as a flat, rigid body separated by a distance $\delta$ from the highest points of the protrusions. Since most of the interaction energy is localized to the neighborhood of the points of grazing contact, we simplify the calculation of by approximating the $\mathrm{vdW}$ interaction potential $V_{\mathrm{p}}^{\prime}$ of each squared cosine protrusion using the interaction potential of its osculating sphere at the point of grazing contact [Fig. 1(c)]. The error compared to direct use of Eqs. (1) and (4) is discussed in Appendix A for the case of a flat plate. The osculating sphere has a radius of curvature

$$
r=\frac{\lambda^{2}}{2 \pi^{2} b} .
$$

The interaction potential between a sphere and a film, as obtained by superposition using the formula for a sphere close to a half space derived by Hamaker [26], is

$$
V_{\mathrm{s}}^{\prime}(\delta)=-\frac{A r}{6 \delta}+\frac{A r}{6(\delta+d)} \approx-\frac{A r}{6 \delta},
$$

where, again, only the vdW part is included. The subscript "s" indicates that the protrusion is approximated by a sphere. The error introduced by this sphere approximation vanishes when $b / \delta \rightarrow \infty$ (Appendix A).

Equation (12) will be used to find an approximation for the interaction potential for the geometry of Fig. 2(b). First, however, we derive an estimate for the radius of curvature $R$ of the film during peel-off. To this end, we homogenize the interactions of the contact interface. For a flat surface of length $\xi$ and width $w$ at the equilibrium distance $\delta$ from the top of
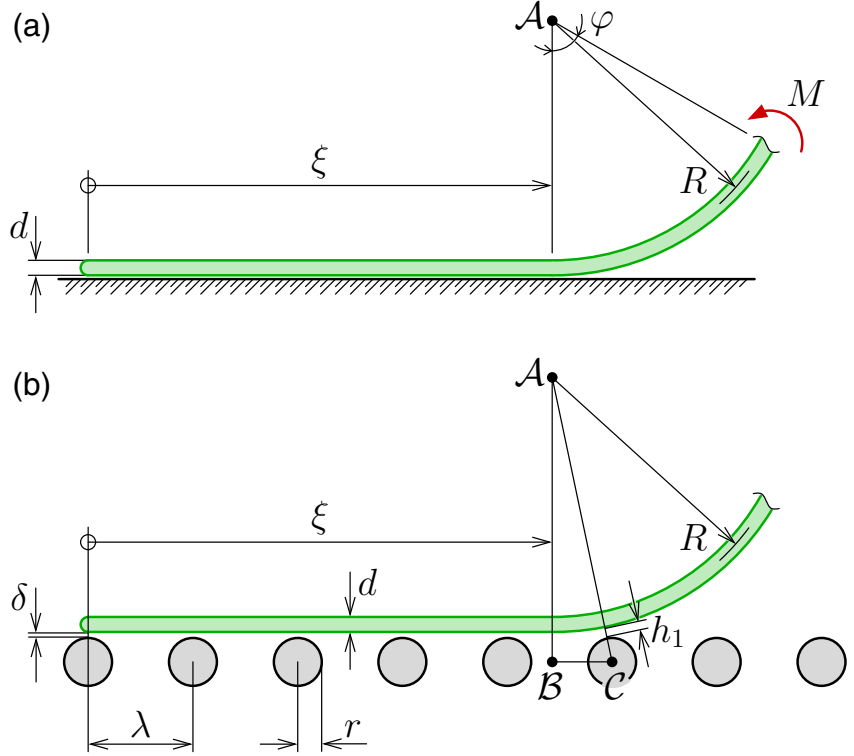

FIG. 2. (Color online) (a) Peel-off using a couple $M$ applied to the edge of the film. (b) Geometry of the model used for estimating the film-surface interaction potential. 
the spheres, we then have

$$
V_{\mathrm{s}}(\xi ; \delta)=-\frac{A r}{6 \delta} \frac{\xi w}{\lambda^{2}},
$$

where $\xi w / \lambda^{2}$ is the number of protrusions covered by the area $\xi w$, allowing for partial spheres. The semicolon in $V_{\mathrm{s}}(\xi ; \delta)$ indicates that $V_{\mathrm{s}}$ is a function of $\xi$, parameterized by $\delta$.

When a film of width $w$ and length $L$ is peeled off of a surface, a certain length $\xi$ of film is adhered, while the midplane of the film bends upward with a typical radius of curvature $R$ at the peeled-off edge [Fig. 2(a)]. For the purpose of estimating this radius $R$, we consider interaction with the protrusions of the flat, rectangular part of the film in contact only, while the bent part is assumed to be distant enough that its contribution is negligible, so that $V_{\mathrm{s}}$ according to Eq. (13) can be used for the interaction potential. The estimated value of $R$ will be used in a somewhat more accurate approximation of $V_{\mathrm{s}}$ later in Eq. (18). Thus, consider a slow, quasistatic peel-off process by the application of a couple $M$ at one edge of the film [Fig. 2(a)]. Modeling the film as a Kirchhoff plate, the relation between this couple and the curvature $1 / R$ of the plate is given by $M=D w / R$. For a thin film of total length $L$, we have $L=\xi+R \varphi$, where $\varphi$ is the angle of the circular arc formed by the curved region of the film. The elastic bending energy of this curved region, modeled as a Kirchhoff plate, is

$$
W_{\mathrm{b}}=\frac{M^{2} R \varphi}{2 D w}=\frac{D w \varphi}{2 R} .
$$

For a quasistatic process, the structure is in equilibrium. Assuming that the kinematics of the process is described fully by specifying $\varphi$ and $\xi(\varphi)$, the condition for minimum total potential energy is

$$
d \Phi=-d U+d W_{\mathrm{b}}+d V_{\mathrm{s}}=0,
$$

with $d U=M d \varphi$ the differential work. In the present case,

$$
-M d \varphi+\frac{d W_{\mathrm{b}}}{d \varphi} d \varphi+\frac{d V_{\mathrm{s}}}{d \xi} \frac{d \xi}{d \varphi} d \varphi=0,
$$

which, with $d \xi / d \varphi=-R$, evaluates to

$$
R=\sqrt{\frac{6 \pi^{2} D \delta b}{A}} .
$$

This radius was derived for a peeling by the application of a couple, but serves herein as an estimate for the radius of curvature of the film for the general peeling geometry. Note that this calculation of $R$ is performed under the assumption of a constant $R$ during the peel-off process, so that the change in elastic strain energy is due to a change in length of the bent part, rather than to a change in the curvature of the plate. Also note that while there might be no compelling reason to prefer a Kirchhoff plate over a Bernoulli beam in the present calculation, we want to be consistent with the assumptions in Sec. II C.

To calculate the interaction potential for the geometry of Fig. 2(b), we assume that the film has a flat part and a part bent into a circular arc with the radius $R$ calculated above. It is also assumed that the interactions are localized so that the potential is a sum of contributions given by Eq. (12). The film adheres to the underlying surface within a region $0<x<\xi$, $0<y<w$, where the width $w$ of the film is a multiple of $\lambda$, and the radius of curvature near $\xi$ is taken to be $R$ [Fig. 2(b)]. The total interaction potential is approximated by

$$
V(\xi)=\frac{w}{\lambda}\left[\left\lfloor\frac{\xi}{\lambda}\right\rfloor V_{\mathrm{s}}^{\prime}(\delta)+\sum_{j=1}^{\infty} V_{\mathrm{s}}^{\prime}\left(h_{j}\right)\right],
$$

where $\lfloor x\rfloor$ is the largest integer smaller than or equal to $x$, and $h_{j}$ is the separation between the film and the $j$ th line of spheres found in the region $x>\xi$. In fact, $V_{\mathrm{s}}^{\prime}(h)$ is valid only for $h \ll R$. However, the contributions from far-away spheres are assumed to be negligible. Applying Pythagoras's theorem to the triangle $\mathcal{A B C}$ in Fig. 2(b) gives $h_{1}$, and analogous considerations for $j>1$ give

$$
\begin{aligned}
h_{j}= & \sqrt{\left[R+\frac{d}{2}+r+\delta\right]^{2}+\left[\lambda\left(\left\lfloor\frac{\xi}{\lambda}\right\rfloor+j\right)-\xi\right]^{2}} \\
& -R-\frac{d}{2}-r \\
\approx & {\left[\frac{\lambda^{2}}{2 R \delta}\left(\left\lfloor\frac{\xi}{\lambda}\right\rfloor-\frac{\xi}{\lambda}+j\right)^{2}+1\right] \delta, }
\end{aligned}
$$

where the last approximation is obtained by assuming that $d$ and $\lambda$ are much greater than $\delta$ and much smaller than $R$.

Inserting Eq. (19) into Eq. (18), while using the definitions of $r$ and $V_{\mathrm{s}}^{\prime}$, gives

$$
\frac{V(\xi)}{V_{0}}=-\left\lfloor\frac{\xi}{\lambda}\right\rfloor-\sum_{j=1}^{\infty}\left[\frac{\lambda^{2}}{2 R \delta}\left(\left\lfloor\frac{\xi}{\lambda}\right\rfloor-\frac{\xi}{\lambda}+j\right)^{2}+1\right]^{-1},
$$

where $V_{0}=A w \lambda / 12 \pi^{2} \delta b$ is the unsigned contribution to the interaction potential from one transverse line of protrusions. This normalized potential $V(\xi) / V_{0}$ is plotted against the nondimensional adhered length $\xi / \lambda$ in Fig. 3 for different values of $\lambda^{2} / R \delta=\left\{10^{0}, 10^{1}, 10^{2}, 10^{3}\right\}$. For small values of $\lambda^{2} / R \delta \ll 1$, the normalized potential is simply a ramp function, while for larger vales $\lambda^{2} / R \delta \gg 1$, the potential becomes a staircase-like function [Fig. 3].

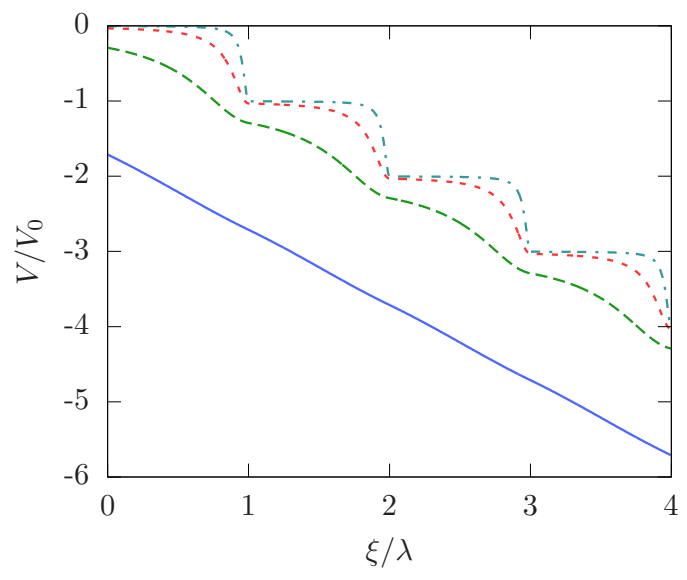

FIG. 3. (Color online) The normalized potential as a function of the nondimensionalized adhered length for different values of $\lambda^{2} / R \delta$ : $10^{0}$ (solid blue line), $10^{1}$ (dashed green line), $10^{2}$ (dotted red line), and $10^{3}$ (dash-dotted cyan line). 


\section{E. Phase diagram of the adhesion system}

Based on the parameters $r_{\mathrm{f}}$ and $\lambda^{2} / R \delta$, three distinctly different types of adhesive behavior can be identified:

(1) Complete contact adhesion. For $r_{\mathrm{f}} \gg 1$, the film follows the topography of the surface, producing an interaction potential $V(\xi)=-\gamma_{\text {eff }} w \xi$. This is the case studied in Sec. II C. The contribution from the kink in the film where it loses contact with the surface is neglected.

(2) Partial contact adhesion. For $r_{\mathrm{f}} \ll 1$ and $\lambda^{2} / R \delta \ll 1$, the film contacts the tips of the surface protrusions, but the small curvature of the film gives a smooth interaction potential $V(\xi)=-V_{0} \xi / \lambda-V_{R}$, where $V_{R}$ denotes the constant contribution from the peel-off region. This is the essentially straight, solid line in Fig. 3.

(3) Glassy adhesion. For $r_{\mathrm{f}} \ll 1$ and $\lambda^{2} / R \delta \gg 1$, the adhesion energy is localized to microscopic areas of contact. The potential decreases in sudden jumps as the film is peeled off, with an interaction potential given by Eq. (20). This corresponds to the staircase curves in Fig. 3.

The elastic energy stored in the curved region of the film during peel-off is not included in the interaction potential, since this elastic energy is created upon initiation of the peel-off and liberated at the end of the peel-off process.

The term glassy adhesion derives from the the glassy dynamics exhibited by the crack front in this regime. Taking $\lambda^{2} / R \delta=1$, the solid line in Fig. 3, as defining the border between partial contact and glassy behavior, this border can be written

$$
r_{\mathrm{f}}=c_{\mathrm{p}}(b / \delta), \quad c_{\mathrm{p}}(b / \delta)=\frac{\delta}{2 \pi^{3} b},
$$

by the use of Eqs. (10) and (17), where the function $c_{\mathrm{p}}$ is specific to the square cosine pattern. Using Eq. (21) together with the condition $r_{\mathrm{f}}>1$ defining complete contact behavior, we can construct a phase diagram for thin-film adhesion, which is illustrated schematically in Fig. 4.

\section{ADHESIVE FAILURE}

We investigate adhesive failure driven by applying a peeloff force to one edge of a partially adhered film. We derive the

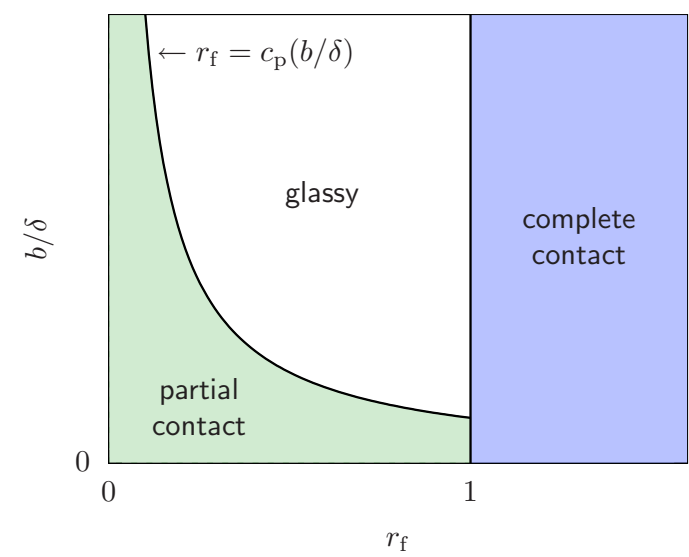

FIG. 4. (Color online) Phase diagram of thin-film adhesion to a surface with a grid of protrusions.

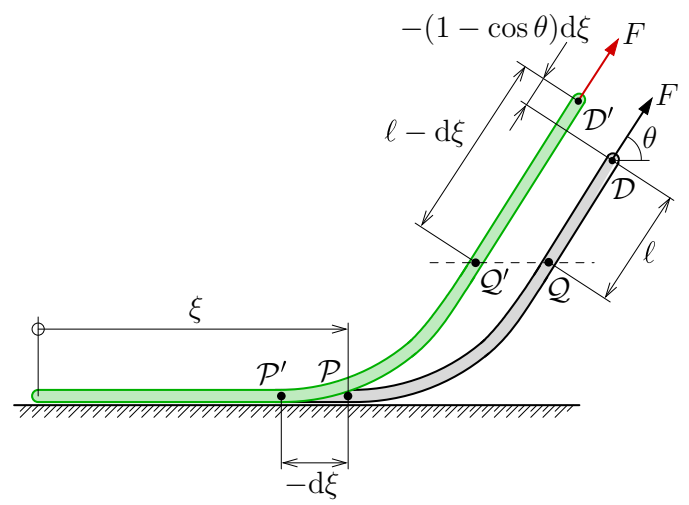

FIG. 5. (Color online) Peel-off geometry for a force $F$ applied to the edge of an adhered film. The suspended part of the film traces an $\operatorname{arc} \mathcal{P} \mathcal{Q D}$ in one instance. When a crack propagates a distance $-d \xi$, the film assumes a new shape $\mathcal{P}^{\prime} \mathcal{Q}^{\prime} \mathcal{D}^{\prime}$.

potential for such a system, and consider the thermally assisted dynamics of failure.

\section{A. Potential of the film during peel-off}

Consider a thin film that is partially adhered to a patterned surface while being peeled off by applying a force $F$ to the free edge of the film [15], so that the force makes an angle $\theta$ with the plane of contact [Fig. 5]. The width of the film is $w$ and a length $\xi$ of film is adhered. The point at which the film loses contact with the surface is denoted by $\mathcal{P}$. It is assumed that there is a point $\mathcal{Q}$ a distance $\ell$ from the free end $\mathcal{D}$ of the film, such that $\mathcal{P Q}$ forms an arc along the film, whose length and shape are preserved during the peel-off process to a good approximation. Moreover, $\mathcal{Q}$ is chosen so that the part $\mathcal{Q D}$ can be considered to be flat. These assumptions regarding the peel-off geometry are implicit in the work of Kendall [15]. With this geometry [Fig. 5], the differential work carried out by the applied force is

$$
d U=-F(1-\cos \theta) d \xi .
$$

The elastic bending energy $W_{\mathrm{b}}$ stored in the $\operatorname{arc} \mathcal{P} \mathcal{Q}$ is unchanging:

$$
d W_{\mathrm{b}}=0 .
$$

The energy of the essentially flat part $\mathcal{Q D}$ is taken as the stretching energy of a Kirchhoff plate in uniaxial tension (Appendix B):

$$
W_{\mathrm{s}}=\frac{\left(1-v^{2}\right) F^{2}}{2 E_{\mathrm{Y}} w d} \ell \Rightarrow d W_{\mathrm{s}}=-\frac{\left(1-v^{2}\right) F^{2}}{2 E_{\mathrm{Y}} w d} d \xi
$$

The potential $\Phi$ of this system subject to changes in the adhered length $\xi$ is chosen so that

$$
\begin{aligned}
d \Phi & =-d U+d W_{\mathrm{b}}+d W_{\mathrm{s}}+d V \\
& =F(1-\cos \theta)\left[1-\frac{\left(1-v^{2}\right) F}{2(1-\cos \theta) E_{\mathrm{Y}} w d}\right] d \xi+d V \\
& =Q d \xi+d V,
\end{aligned}
$$

when $\theta>0$. Here, we define the generalized force as

$$
Q=F(1-\cos \theta)\left[1-\frac{\left(1-v^{2}\right) F}{2(1-\cos \theta) E_{\mathrm{Y}} w d}\right] .
$$




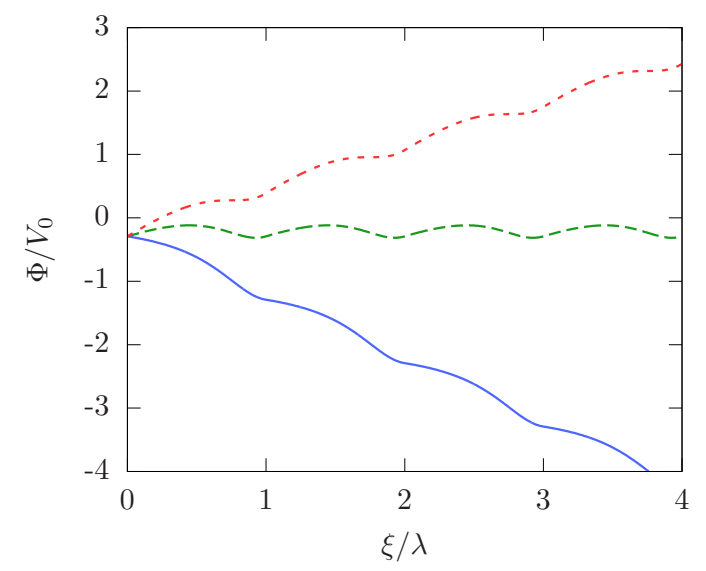

FIG. 6. (Color online) Normalized total energy as a function of nondimensional adhesion length for $\lambda^{2} / R \delta=10$ and different values of the nondimensional, generalized force $Q \lambda / V_{0}: 0$ (solid blue line), 1 (dashed green line), 1.68 (dotted red line).

The elastic energy term enters as a reduction of the applied generalized force. This elastic term only becomes non-negligible in two cases, as previously observed [15]:

(1) When the engineering stress $F / w d$ becomes comparable to $E_{\mathrm{Y}}$, which is possible for highly extensible elastomers.

(2) When the angle $\theta$ is small.

Using Eq. (25) and neglecting any effect of gravitation, we put

$$
\Phi(\xi)=Q \xi+V(\xi),
$$

$Q$ being a dead load.

For complete contact adhesion, the interaction potential is $V(\xi)=-\gamma_{\text {eff }} w \xi$, as discussed in Sec. II E, yielding a potential $\Phi=\left(Q-\gamma_{\mathrm{eff}} w\right) \xi$. Adhesive failure occurs when the gradient of the potential becomes positive, that is, when $Q>\gamma_{\mathrm{eff}} w$, at which point some dynamic process drives $\xi$ toward zero. Similarly, for partial contact adhesion with $V=-V_{0} \xi / \lambda-$ $V_{R}$, we have $\Phi=\left(Q-V_{0} / \lambda\right) \xi-V_{R}$, and adhesive failure occurs when $Q>V_{0} / \lambda$.

In the glassy regime of adhesion, the potential energy is not linear in $\xi$. Then, if thermal fluctuations are neglected, adhesive failure is understood as an instability arising from the elimination of local minima of $\Phi$ when the applied generalized force reaches a critical limit. The minima of the potential are the solutions to

$$
\left\{\begin{array} { l } 
{ \frac { d \Phi } { d \xi } = 0 } \\
{ \frac { d ^ { 2 } \Phi } { d \xi ^ { 2 } } > 0 }
\end{array} \Leftrightarrow \left\{\begin{array}{c}
Q+\frac{d V}{d \xi}=0 \\
\frac{d^{2} V}{d \xi^{2}}>0
\end{array}\right.\right.
$$

These minima are eliminated when $Q>-d V / d \xi$ for all $\xi$, corresponding to adhesive failure, or when $Q<-d V / d \xi$, corresponding to an increasing adhesion area. Thus, adhesive failure will occur when

$$
Q>\max _{\xi>0}\left(-\frac{d V}{d \xi}\right)
$$

Figure 6 illustrates how $\Phi$ is affected by applying different nondimensional generalized forces $Q \lambda / V_{0} \in\{0,1,1.68\}$ to a glassy adhesion system with $\lambda^{2} / R \delta=10$ and $V(\xi)$ as in Eq. (20). In this instance and with a zero-temperature assumption, a force $Q \lambda / V_{0}=1.68$ is required to achieve the

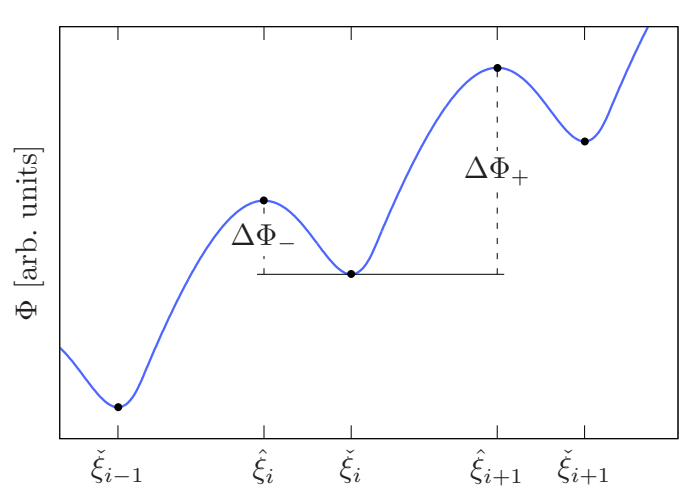

FIG. 7. (Color online) Energy landscape of a glassy adhesive system with $\lambda^{2} / R \delta=10$ and $Q \lambda / V_{0}=1.20$. Local extrema are indicated by points.

condition for adhesive failure, $d \Phi / d \xi>0$ for all $\xi$. Thus, the force required for glassy adhesive failure is typically greater than that of partial contact adhesive failure. The required nondimensional force grows with $\lambda^{2} / R \delta$.

\section{B. Thermally activated adhesive failure}

The energy landscape $\Phi(\xi)$ formed by the glassy adhesive system [Fig. 6] includes local minima, that is, metastable states, for some intermediate values of the generalized force $Q$. Since we consider a nanoscale adhesion system, well within the colloidal regime, and relatively weak vdW attraction, it is reasonable to expect thermally activated state transitions between these metastable states. In this respect, the situation is analogous to the forced motion of a liquid drop across a rough or chemically heterogeneous surface. In that case, the three-phase contact line becomes trapped at local energy minima. At molecular scales, when the energy minimum is in the order of several $k_{\mathrm{B}} T$, with $k_{\mathrm{B}}$ the Boltzmann constant and $T$ the absolute temperature, contact line depinning transitions are assisted by thermal activation, as described by the molecular kinetics theory (MKT) [27]. Such intermittent contact line motion has been demonstrated for many wetting systems [28-30].

Consider the potential $\Phi(\xi)$ of a glassy film adhesion system, and an applied, generalized force $Q$ in the intermediate range for which $\Phi$ becomes nonmonotonic. Metastable states are found at the coordinates $\check{\xi}_{i}$ giving local minima of $\Phi$, enumerated so that $\breve{\xi}_{i}<\breve{\xi}_{i+1}$. So-called transition states are found at the coordinates $\hat{\xi}_{i}$ giving local maxima of $\Phi$, enumerated so that $\breve{\xi}_{i-1}<\hat{\xi}_{i}<\breve{\xi}_{i}$. We define the forward and backward transition energy barriers as

$$
\begin{aligned}
& \Delta \Phi_{+}=\Phi\left(\hat{\xi}_{i+1}\right)-\Phi\left(\check{\xi}_{i}\right), \\
& \Delta \Phi_{-}=\Phi\left(\hat{\xi}_{i}\right)-\Phi\left(\check{\xi}_{i}\right),
\end{aligned}
$$

respectively, as depicted in Fig. 7. We adopt the views of transition state theory [12]. That is, the system becomes trapped in a local minimum $\breve{\xi}_{i}$ and fluctuates with some frequency $\omega$ in the basin of the minimum due to stochastic impulses from collisions with molecules of the surrounding medium. The energy of the system is assumed to follow the Boltzmann distribution. Hence, the probability that the 


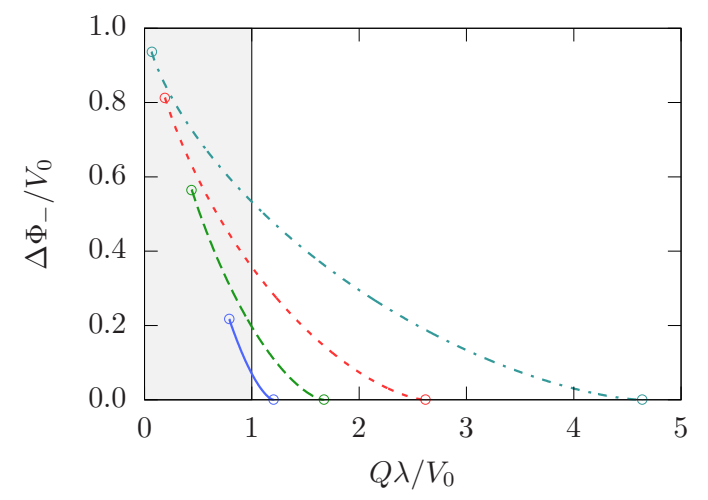

FIG. 8. (Color online) Backward energy barrier as a function of applied force for different values of $\lambda^{2} / R \delta: 3$ (solid blue line), 10 (dashed green line), 30 (dotted red line), and 100 (dash-dotted cyan line). Adhesive failure occurs when $Q \lambda / V_{0}>1$. Instantaneous failure occurs when $\Delta \Phi_{-}$reaches zero.

energy exceeds $\Phi\left(\breve{\xi}_{i}\right)+E$ at any given time is $\exp \left(-E / k_{\mathrm{B}} T\right)$. Forward transitions, that is, transitions from the $i$ th metastable state to the $i+1$ st metastable state, thus occur at a rate

$$
k_{+}=\omega \exp \left(-\frac{\Delta \Phi_{+}}{k_{\mathrm{B}} T}\right) .
$$

Similarly, backward transitions from the $i$ th state to the $i-1$ st state occur at a rate

$$
k_{-}=\omega \exp \left(-\frac{\Delta \Phi_{-}}{k_{\mathrm{B}} T}\right) .
$$

Each forward transition increases and each backward transition decreases the adhered length by $\lambda$. This means that the timeaverage propagation velocity of the crack (in the negative $\xi$ direction) during adhesive failure becomes

$$
v=-\lambda\left(k_{+}-k_{-}\right)
$$

and macroscopic adhesive failure occurs when $k_{-}>k_{+}$, that is, when $Q \lambda / V_{0}>1$. However, this failure will not be instantaneous; in fact, it may not even occur within experimental time-scales if the energy barrier is very large, $\Delta \Phi_{-} / k_{\mathrm{B}} T \gg 1$.

In a prototypical glassy adhesion failure mechanism, $k_{-} \gg$ $k_{+}$due to the exponential dependence of the rate on the energy barriers. This leads to the approximation

$$
v=\lambda k_{-}=\lambda \omega \exp \left(-\frac{\Delta \Phi_{-}}{k_{\mathrm{B}} T}\right) .
$$

Here $\Delta \Phi_{-}$depends on the applied force $Q$, and this relation is investigated numerically. The nondimensional energy barrier $\Delta \Phi_{-} / V_{0}$ is plotted against nondimensional force $Q \lambda / V_{0}$ for different values of $\lambda^{2} / R \delta=\{3,10,30,100\}$ in Fig. 8. This relation can be used to find the velocity of the crack front, rewriting Eq. (34) as

$$
\frac{v}{\lambda \omega}=\exp \left(-\frac{V_{0}}{k_{\mathrm{B}} T} \frac{\Delta \Phi_{-}}{V_{0}}\right) .
$$

The nondimensional rate $v / \lambda \omega$ is thus controlled by three nondimensional parameters of the system:

$$
\frac{V_{0}}{k_{\mathrm{B}} T}, \quad \frac{\lambda^{2}}{R \delta}, \text { and } \frac{Q \lambda}{V_{0}} \text {. }
$$

\section{DISCUSSION}

\section{A. Corrugated surfaces}

The derivations above are particular to surfaces with a regular grid of protrusions. However, we may regard the derivations as a template that can be employed for any regular surface topography, i.e., a corrugated surface [Fig. 1(b)]:

$$
f(x, y)=b \cos ^{2} \frac{\pi x}{\lambda} .
$$

Direct computation of the elastic energy per unit area for a film conforming to this corrugation yields $W_{\mathrm{e}} / A_{S}=\pi^{4} D b^{2} / \lambda^{4}$, which happens to be the same expression as for the grid of protrusions. Hence, the expression for the flexibility ratio in Eq. (10) holds for the corrugated surface as well.

The radius of curvature $r$ of the tips of the corrugation is given by Eq. (11). The contacts between a semirigid film and the corrugated surface can, similarly to Sec. IID, be approximated using a raster of osculating cylinders of radius $r$ a distance $\delta$ from the film surface [Fig. 1(d)]. Treating this geometry analogously to the grid of spheres to estimate the radius of curvature of the film near the peel-off line, we find (Appendix C)

$$
R=\sqrt{\frac{12 \pi D \delta^{3 / 2} b^{1 / 2}}{A}} .
$$

Moreover, the potential of a film being peeled off from a corrugated surface is computed in Appendix $\mathrm{C}$, and it is shown that this potential becomes a ramp function when $\lambda^{2} / R \delta \ll 1$ and staircase-like when $\lambda^{2} / R \delta \gg 1$. This is the same behavior as that of the grid of protrusions.

For the corrugated surface, the border defined by $\lambda^{2} / R \delta=$ 1 between partial contact and glassy adhesion becomes

$$
r_{\mathrm{f}}=c_{\mathrm{c}}(b / \delta), \quad c_{\mathrm{c}}(b / \delta)=\frac{\delta^{3 / 2}}{\pi^{4} b^{3 / 2}} .
$$

This expression deviates slightly from that of the grid of protrusions. However, it is still possible to identify the complete contact adhesion, partial contact adhesion and glassy adhesion in a phase diagram (not shown), demonstrating the generality of those phases of adhesion.

\section{B. Gecko spatulae}

As described by Autumn and Peattie [19], a long history of experimental investigations in the literature have identified $\mathrm{vdW}$ interaction as an important mechanism for the gecko adhesion. The adhesive strength is enhanced by humidity [31], but this humidity dependence may be due to modification of the $\mathrm{vdW}$ attraction rather than to capillary effects [31]. It has been shown experimentally by direct imaging that the spatula of the gecko's adhesion system adheres to a flat surface similarly to a film adhering to a surface [17]. Using a thin film to model spatula adhesion has also been advocated elsewhere $[32,33]$. In this section, we investigate the adhesion of gecko spatulae to 
a patterned soda lime glass surface, assuming that the surface undulations can be regarded as an idealized surface roughness, as commonly assumed in previous modeling work [20,21]. We also neglect viscoelastic effects, not because they are necessarily insignificant, but for the purpose of isolating the contribution associated with the theoretical frame developed in this work.

The setae and spatulae of the gecko are composed of $\beta$-keratin [34], which has a Young's modulus of $E_{\mathrm{Y}}=$ 1.6 GPa, as experimentally determined for the seta shaft [35]. The spatulae are about $w=200 \mathrm{~nm}$ wide at their widest edge [36,37], have a thickness of $d \in[5,10] \mathrm{nm}$, and become thinner toward the tip, as demonstrated by direct imaging of the spatula cross section [20,38,39].

The strength of the keratin-air-glass vdW interactions depends on the Hamaker constant $A=7.9 \times 10^{-20} \mathrm{~J}$ (Appendix D) and on the equilibrium separation $\delta$ between the keratin and the substrate, at which Pauli repulsion balances the vdW attraction. From peel-off experiments for a single gecko spatula adhering to a flat glass surface, it has been determined that $\gamma \approx 50 \mathrm{~mJ} / \mathrm{m}^{2}$ [38]. Using this value with Eq. (3) gives $\delta=0.20 \mathrm{~nm}$, which is also in the order of magnitude expected for Pauli repulsion.

We consider a spatula of thickness $d=5 \mathrm{~nm}$, width $w=$ $200 \mathrm{~nm}$ and adhered length $L=100 \mathrm{~nm}$, together with vdW interaction parameters discussed above, and map the phase diagram [Fig. 4] onto the $\lambda-b$ plane [Fig. 9]. This mapping reveals the regime of glassy adhesion. It is now possible to plot the velocity of the crack during adhesive failure against the applied force for each choice of surface parameters $(\lambda, b)$ in the glassy regime.

As an example, we choose the surface parameters $\lambda=$ $10 \mathrm{~nm}$ and $b=4 \mathrm{~nm}$, and plot the backward energy barrier $\Delta \Phi_{-}$as a function of applied force $Q$ [Fig. 10(a)]. For this choice of parameters, the energy barrier of the glassy regime is in the order of a few tens of $k_{\mathrm{B}} T$, which, as a chemist's rule of thumb, corresponds to kinetics occurring at experimental time scales. The generalized force required to initiate adhesive failure is around $0.2 \mathrm{nN}$. Owing to the relatively high aspect ratio protrusions, this is somewhat lower than published measurements of the adhesion force of the gecko spatula against a flat cover glass slip, which is around $10 \mathrm{nN} \mathrm{[38].}$

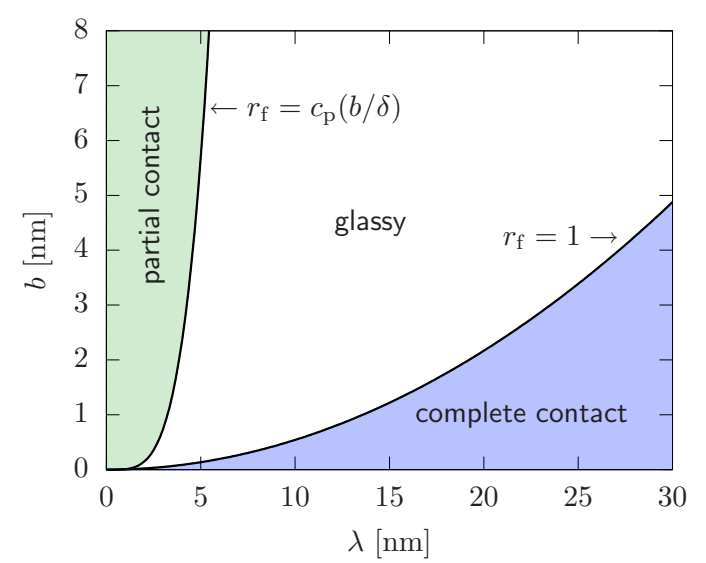

FIG. 9. (Color online) Regimes of different types of adhesion for the gecko spatula adhesion system.
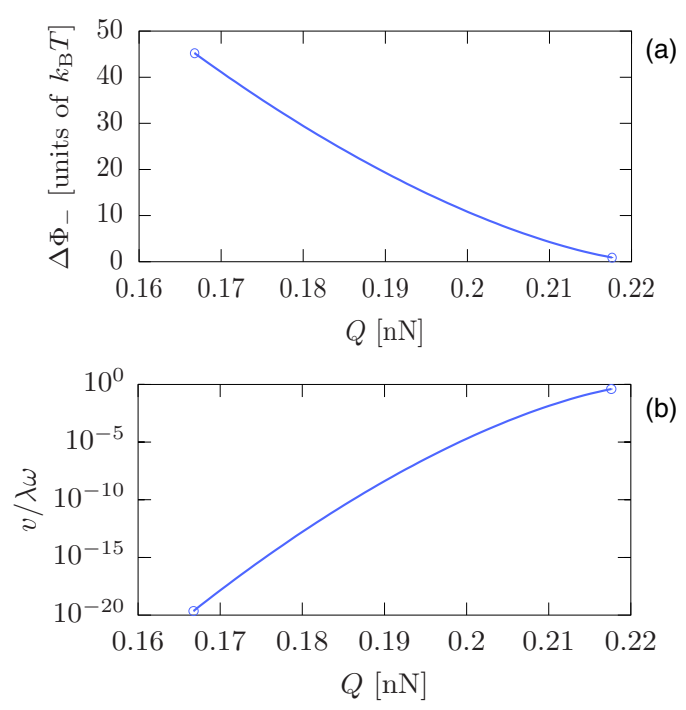

FIG. 10. (Color online) (a) Backward energy barrier as a function of force for spatula adhesion on a glass surface with $\lambda=10 \mathrm{~nm}$ and $b=4 \mathrm{~nm}$. (b) Force-rate relation for spatula adhesion.

The nondimensional crack velocity is plotted against the applied force in Fig. 10(b). It is observed that the rate of the adhesive failure increases exponentially with the applied force. Clearly, glassy adhesion is most relevant for the case of static fatigue of the adhesive interface, when a constant force is applied and it is more realistic to neglect viscoelastic effects. In this case the applied force has a huge effect on the lifetime of the adhesive contact. Conversely, for a constant-rate peel-off process, the peel-off rate has a relatively small effect on the adhesion force. In the given example, the adhesion force increases by $30 \%$ from smallest to greatest peel-off rate [Fig. 10(b)].

\section{CONCLUSIONS}

The process of peeling off a thin film adhering to a patterned surface by vdW attraction has different characteristics depending on the stiffness of the film, the topography of the surface and the strength of vdW interactions. We define the flexibility ratio $r_{\mathrm{f}}$ as the ratio between the $\mathrm{vdW}$ interaction energy for a film adhering to a flat surface, and the elastic energy stored in a Kirchhoff plate that conforms to the undulating surface. This dimensionless number is used to distinguish between different regimes of adhesion.

A film of low bending stiffness, with $r_{\mathrm{f}} \gg 1$, follows the surface undulations and remains in grazing contact with the surface across the nominal contact area. In this case, the situation is similar to that of a film contacting a flat surface, but, as previously suggested [20,21], the elastic energy stored in the film act to decrease the effective interaction energy. A sufficiently stiff film, $r_{\mathrm{f}} \ll 1$, remains flat while contacting the undulating surface and remains suspended on top of the protrusions. Because vdW interactions decrease rapidly with the distance $\rho$ between the interacting material points, the potential scales as $\rho^{-6}$, the interaction energy becomes localized to microscopic areas of contact. For an intermediate range of flexibility ratios, e.g., when $c_{\mathrm{p}}(b / \delta) \ll r_{\mathrm{f}} \ll 1$ for 
a square grid of protrusions, the potential of the film during peel-off includes local minima similar to those of a three-phase contact line on a heterogeneous surface. For a nanosize film contacting a micropatterned surface, the energy barriers between these local minima is in the order of several $k_{\mathrm{B}} T$. In this regime of glassy adhesion, the crack front progresses through thermally activated transitions between the metastable states. Applying a peel-off force reduces the energy barriers and thus increases the failure rate dramatically: the crack front velocity increases exponentially with force.

In any physical adhesive system, the rate effect predicted herein for glassy adhesive failure competes with other rate effects, including the viscoelasticity or plasticity of the film, hygroscopic effects, and others. Since the force is logarithmic in the rate for glassy adhesive failure, other rate effects are likely to dominate rapid processes of rate-controlled failure. For the same reason, the glassy adhesion effect is more likely to dominate slow adhesive failure due to the application of a static load.

\section{ACKNOWLEDGMENT}

The authors thank Dr J. Sprakel for useful discussions.

\section{APPENDIX A: ERROR FROM SPHERE APPROXIMATION OF PROTRUSIONS}

Consider a half space with a surface topography given by Eq. (4), representing a square grid of protrusions, with $b$ the protrusion height and $\lambda$ their separation. A flat, rigid film of thickness $d$ is parallel to this surface, separated by a distance $\delta$ from the tips of the protrusions of the surface. Then we may choose a Cartesian coordinate system such that the half space occupies the region $\Omega=\{\vec{x}: z<f(x, y)-b-\delta\}$, with $\vec{x}=(x, y, z)$ the position vector, and the film occupies $\Omega^{\prime}=\left\{\vec{x}^{\prime}: 0<z^{\prime}<d\right\}$.

From Eq. (1), the differential London-van der Waals (vdW) interaction potential $d V$ between a volume element $d x d y d z$ in $\Omega$ and the film is

$$
\begin{aligned}
d V & =\left(-\frac{A}{\pi^{2}} \int_{\Omega^{\prime}} \frac{d x^{\prime} d y^{\prime} d z^{\prime}}{\left|\vec{x}-\vec{x}^{\prime}\right|^{6}}\right) d x d y d z \\
& =-\frac{A}{6 \pi}\left[\frac{1}{(z-d)^{3}}-\frac{1}{z^{3}}\right] d x d y d z
\end{aligned}
$$

with $A$ the Hamaker constant. This result is used to compute the vdW interaction potential $V_{\mathrm{p}}^{\prime}$ for a single protrusion. We integrate across a unit cell $\Omega_{\mathrm{p}}=\{\vec{x}: \vec{x} \in \Omega \wedge x, y \in[0, \lambda]\}$ giving

$$
\begin{aligned}
V_{\mathrm{p}}^{\prime}= & \int_{\Omega_{\mathrm{p}}} d V=-\frac{A}{6 \pi} \int_{\Omega_{\mathrm{p}}}\left[\frac{1}{(z-d)^{3}}-\frac{1}{z^{3}}\right] d x d y d z \\
= & \frac{A}{12 \pi} \int_{0}^{\lambda} \int_{0}^{\lambda} \frac{d x d y}{[f(x, y)-b-\delta-d]^{2}} \\
& -\frac{A}{12 \pi} \int_{0}^{\lambda} \int_{0}^{\lambda} \frac{d x d y}{[f(x, y)-b-\delta]^{2}},
\end{aligned}
$$

where the prime indicates that this is the interaction between the film and a single protrusion.

Next, we will investigate an approximation of the interaction potential from a single protrusion $V_{\mathrm{p}}^{\prime}$. Since $V_{\mathrm{p}}^{\prime}$ is

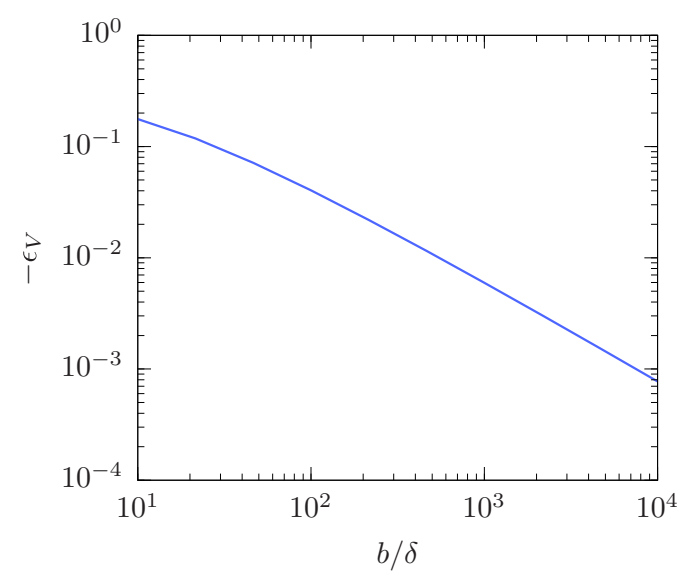

FIG. 11. (Color online) The relative error in the interaction potential due to the approximation of surface protrusions by spheres.

dominated by the contribution from the peak of the protrusion, the interaction potential for the squared cosine protrusions is approximated by that of osculating spheres with radius equal to the radius of curvature $r$ of the protrusions at the contact points:

$$
r=\frac{\lambda^{2}}{2 \pi^{2} b} .
$$

The interaction potential between a sphere and the film, as obtained by superposition using the formula for a sphere close to a half space [26], is

$$
V_{\mathrm{s}}^{\prime}(\delta)=-\frac{A r}{6 \delta}+\frac{A r}{6(\delta+d)} \approx-\frac{A r}{6 \delta} .
$$

The subscript "s" indicates that the protrusion is approximated by a sphere.

The relative error in the interaction potential introduced by this sphere approximation is given by

$$
\begin{aligned}
\epsilon_{V} & =\frac{V_{\mathrm{s}}^{\prime}}{V_{\mathrm{p}}^{\prime}}-1 \\
& \approx \frac{\lambda^{2}}{\pi b \delta}\left\{\int_{0}^{\lambda} \int_{0}^{\lambda} \frac{d x d y}{[f(x, y)-b-\delta]^{2}}\right\}^{-1}-1, \\
& =\pi \frac{b}{\delta}\left\{\int_{0}^{\pi} \int_{0}^{\pi} \frac{d s d t}{\left[1-\cos ^{2} s \cos ^{2} t+\delta / b\right]^{2}}\right\}^{-1}-1,
\end{aligned}
$$

where the approximation holds for $d \gg \delta$ and a substitution $s=\pi x / \lambda$ and $t=\pi y / \lambda$ was made. It is shown by numerical evaluation in Fig. 11 that $\epsilon_{V}<0$ and that $\epsilon_{V}$ vanishes when $\delta \ll b$.

\section{APPENDIX B: KIRCHOFF PLATE IN UNIAXIAL TENSION}

Consider a flat plate of length $\ell$, width $w$, thickness $d$, with Young's modulus $E_{\mathrm{Y}}$ and Poisson's ratio $v$. We introduce a Cartesian coordinate frame, with $x$ in the length direction and $z$ in the thickness direction. When loaded with a force $F$ in uniaxial tension in the length direction, the stretching energy 
of a plate becomes [40, p. 243]

$$
W_{\mathrm{s}}=\int_{S} \frac{E_{\mathrm{Y}} d}{2\left(1-v^{2}\right)}\left(\frac{\partial u}{\partial x}\right)^{2} d x d y,
$$

with $u$ the displacement field and $S$ the $x y$ domain of the plate. The force per unit width of film is [40, p. 245]

$$
\frac{F}{w}=\frac{E_{\mathrm{Y}} d}{\left(1-v^{2}\right)} \frac{\partial u}{\partial x} \text {. }
$$

Taken together, this yields

$$
W_{\mathrm{s}}=\frac{\left(1-v^{2}\right) F^{2} \ell}{2 E_{\mathrm{Y}} w d} .
$$

Here, apart from dropping strain energy terms from bending, we have dropped terms involving strain transverse to the loading direction.

\section{APPENDIX C: THIN-FILM ADHESION TO CORRUGATED SURFACES}

The derivation of the interaction potential conducted for a grid of protrusions is repeated here for a corrugated surface, whose topography can be described by

$$
f(x, y)=b \cos ^{2} \frac{\pi x}{\lambda} .
$$

A film of thickness $d$ and plate stiffness $D$ comes into nonconforming contact against this corrugated surface. The $\mathrm{vdW}$ interaction energy becomes localized to the ridges of the corrugation, and we simplify the calculation of the interaction potential by the osculating cylinders of radii

$$
r=\frac{\lambda^{2}}{2 \pi^{2} b}
$$

running in parallel along these ridges.

The vdW interaction potential per unit length of two parallel, infinitely long cylinders of radii $r_{1}$ and $r_{2}$ separated by a distance $H$ is $[23$, p. 254]

$$
\mathcal{V}_{\circ \circ}\left(r_{1}, r_{2}, H\right)=-\frac{A}{24 H^{3 / 2}} \sqrt{\frac{2 r_{1} r_{2}}{r_{1}+r_{2}}} .
$$

The interaction potential per unit length between a cylinder and a half space is obtained as

$$
\mathcal{V}_{\circ \mid}\left(r_{1}, H\right)=\lim _{r_{2} \rightarrow \infty} \mathcal{V}_{\circ \circ}\left(r_{1}, r_{2}, H\right)=-\frac{\sqrt{2} A r_{1}^{1 / 2}}{24 H^{3 / 2}} .
$$

Using the principle of superposition, the interaction potential per unit length for the osculating cylinder and the film becomes

$$
\begin{aligned}
\mathcal{V}_{\mathrm{c}}^{\prime} & =\mathcal{V}_{\text {○| }}(r, \delta)-\mathcal{V}_{\text {○| }}(r, \delta+d) \\
& =-\frac{A \sqrt{2 r}}{24 \delta^{3 / 2}}+\frac{A \sqrt{2 r}}{24(\delta+d)^{3 / 2}} \approx-\frac{A \lambda}{24 \pi b^{1 / 2} \delta^{3 / 2}},
\end{aligned}
$$

where Eq. (C2) was used and it is was assumed that $d \gg \delta$. The subscript "c" indicates that the potential of a corrugation ridge is approximated by a cylinder.

For a flat surface of length $\xi$ and width $w$ at the equilibrium distance $\delta$ from the cylinders, the total interaction potential becomes

$$
V_{\mathrm{c}}(\xi ; \delta)=w \mathcal{V}_{\mathrm{c}}^{\prime} \frac{\xi}{\lambda}=-\frac{A w \xi}{24 \pi b^{1 / 2} \delta^{3 / 2}},
$$

with $\xi / \lambda$ the number of cylinders covered by the film. This allows us to compute an estimate of the radius $R$ of the film in the peel-off zone by solving Eq. (16) for $R$ while inserting $V_{\mathrm{c}}$ instead of $V_{\mathrm{s}}$, giving

$$
R=\sqrt{\frac{12 \pi D \delta^{3 / 2} b^{1 / 2}}{A}} .
$$

The film adheres to the underlying surface within a region $0<x<\xi, 0<y<w$, where it is flat. Near $\xi_{+}$the film is assumed to bend into a circular arc with its axis parallel to the surface ridges and with a radius of curvature $R$. The total interaction potential is approximated by

$$
V(\xi)=w\left[\left\lfloor\frac{\xi}{\lambda}\right\rfloor \mathcal{V}_{\mathrm{c}}^{\prime}(\delta)+\sum_{j=1}^{\infty} \mathcal{V}_{\mathrm{c}}^{\prime}\left(h_{j}\right)\right]
$$

where $h_{j}$, given by Eq. (10) of the main paper, is the separation between the film and the $j$ th cylinder found in the region $x>\xi$. Inserting $h_{j}$ into Eq. (C8), while using the definitions of $r$ and $\mathcal{V}_{\mathrm{c}}^{\prime}$, gives

$$
\frac{V(\xi)}{V_{0}}=-\left\lfloor\frac{\xi}{\lambda}\right\rfloor-\sum_{j=1}^{\infty}\left[\frac{\lambda^{2}}{2 R \delta}\left(\left\lfloor\frac{\xi}{\lambda}\right\rfloor-\frac{\xi}{\lambda}+j\right)^{2}+1\right]^{-3 / 2},
$$

where $V_{0}=-w \mathcal{V}_{\mathrm{c}}^{\prime}(\delta)$ is the unsigned contribution to the interaction from each cylinder. This normalized potential $V(\xi) / V_{0}$ is plotted against the nondimensional adhered length $\xi / \lambda$ in Fig. 12 for different values of $\lambda^{2} / R \delta=\left\{10^{0}, 10^{1}, 10^{2}, 10^{3}\right\}$. For small values of $\lambda^{2} / R \delta \ll 1$, the normalized potential is a ramp function, while for larger vales $\lambda^{2} / R \delta \gg 1$, the potential becomes a staircase-like function [Fig. 12]. This property of the corrugated surface is the same as for the grid of protrusions.

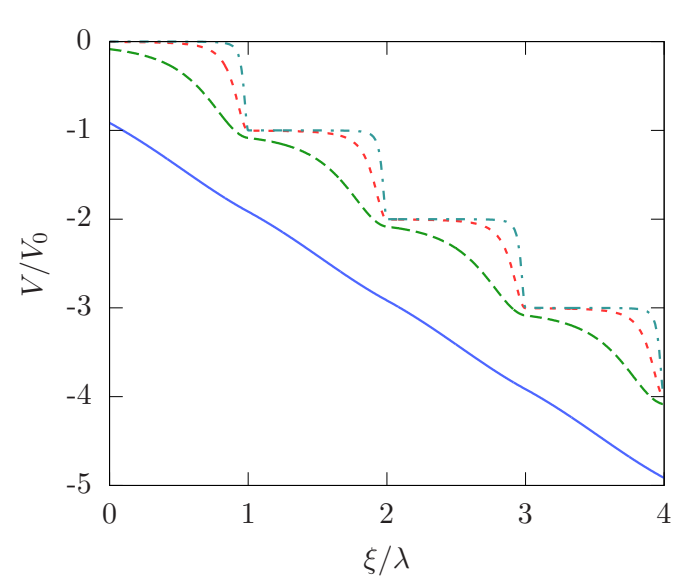

FIG. 12. (Color online) The normalized potential for a film adhering to a corrugated surface as a function of the nondimensionalized adhered length for different values of $\lambda^{2} / R \delta: 10^{0}$ (solid blue line), $10^{1}$ (dashed green line), $10^{2}$ (dotted red line), and $10^{3}$ (dash-dotted cyan line). 


\section{APPENDIX D: HAMAKER CONSTANT FOR KERATIN-GLASS INTERACTIONS}

According to Lifshitz theory, the Hamaker constant for two phases, denoted by indexes 1 and 2 , interacting across a medium with index 3 is [23, p. 260]

$$
\begin{aligned}
A= & \frac{3}{4} k_{\mathrm{B}} T \frac{\left(\epsilon_{1}-\epsilon_{3}\right)\left(\epsilon_{2}-\epsilon_{3}\right)}{\left(\epsilon_{1}+\epsilon_{3}\right)\left(\epsilon_{2}+\epsilon_{3}\right)}+\frac{3 h v_{e}}{8 \sqrt{2}} \\
& \times \frac{\left(n_{1}^{2}-n_{3}^{2}\right)\left(n_{2}^{2}-n_{3}^{2}\right)}{\sqrt{\left(n_{1}^{2}+n_{3}^{2}\right)\left(n_{2}^{2}+n_{3}^{2}\right)}\left(\sqrt{n_{1}^{2}+n_{3}^{2}}+\sqrt{n_{2}^{2}+n_{3}^{2}}\right)},
\end{aligned}
$$

where $k_{\mathrm{B}}$ is the Boltzmann constant, $T$ is the absolute temperature, $h$ is the Planck constant, $v_{e}$ is the absorption frequency for light which is assumed to be the same for all three materials, $\epsilon_{i}$ denotes the relative permittivity and $n_{i}$ is the refractive index of phase $i=1,2,3$. For a keratin-air-glass system, we use the relative permittivity of bovine $\beta$-keratin from the horn, $\epsilon_{1}=20[41,42]$, and the refractive index from tortoise-shell keratin, $n_{1}=1.55$ [43, p. 8]. The surface is taken to be a soda lime glass $\left(\epsilon_{2}=8 ; n_{2}=1.5\right)$ and the medium is air $\left(\epsilon_{3}, n_{3} \approx 1\right)$. All the parameters correspond to room temperature conditions, $T=20^{\circ} \mathrm{C}$. We use $v_{e}=3.0 \times$ $10^{15} \mathrm{~Hz}$, which is a typical value for many different materials [23, p. 263]. For these values, we obtain $A=7.9 \times 10^{-20} \mathrm{~J}$.
[1] E. Arzt, S. Gorb, and R. Spolenak, Proc. Natl Acad. Sci. USA 100, 10603 (2003).

[2] Z. Zhang and T. Li, J. Appl. Phys. 110, 083526 (2011).

[3] A. A. Griffith, Phil. Trans. R. Soc. Lond. A 221, 163 (1921).

[4] J. Sprakel, S. B. Lindström, T. E. Kodger, and D. A. Weitz, Phys. Rev. Lett. 106, 248303 (2011).

[5] S. B. Lindström, T. E. Kodger, J. Sprakel, and D. A. Weitz, Soft Matter 8, 3657 (2012).

[6] K. L. Johnson, K. Kendall, and A. D. Roberts, Proc. R. Soc. Lond. A 324, 301 (1971).

[7] G. C. Sih, P. C. Paris, and G. R. Irwin, Int. J. Fracture Mech. 1, 189 (1965).

[8] N. Gravish, M. Wilkinson, S. Sponberg, A. Parness, N. Esparza, D. Soto, T. Yamaguchi, M. Broide, M. Cutkosky, C. Creton, and K. Autumn, J. R. Soc. Interface 7, 259 (2010).

[9] H. E. Bakker, S. B. Lindström, and J. Sprakel, J. Phys.: Condens. Matter 24, 065103 (2012).

[10] M. K. Chaudhury, J. Phys. Chem. B 103, 6562 (1999).

[11] D. Vella and L. Mahadevan, Langmuir 22, 163 (2006).

[12] H. A. Kramers, Physica 7, 284 (1940).

[13] G. I. Bell, Science 200, 618 (1978).

[14] E. Evans and K. Ritchie, Biophys. J. 72, 1541 (1997).

[15] K. Kendall, J. Phys. D: Appl. Phys. 8, 1449 (1975).

[16] M. K. Johnson and A. P. Russell, J. Anat. 214, 937 (2009).

[17] M. Varenberg, N. M. Pugno, and S. N. Gorb, Soft Matter 6, 3269 (2010).

[18] H. Parsaiyan, F. Barazandeh, M. Parsaiyan, S. M. Rezaei, and M. Safdari, Int. J. Adhes. Adhes. 29, 444 (2009).

[19] K. Autumn and A. M. Peattie, Integr. Comp. Biol. 42, 1081 (2002).

[20] B. N. J. Persson and S. Gorb, J. Chem. Phys. 119, 11437 (2003).

[21] Z. L. Peng and S. H. Chen, Phys. Rev. E 83, 051915 (2011).

[22] I. E. Dzyaloshinkii, E. M. Lifshitz, and L. P. Pitaevskii, Adv. Phys. 10, 165 (1961).

[23] J. N. Israelachvili, Intermolecular and Surface Forces, 3rd ed. (Academic Press, Waltham, MA, 2011).

[24] M. R. Spiegel, Advanced Mathematics for Scientists and Engineers, SI edition (McGraw-Hill, New York, 1980).
[25] L. D. Landau and E. M. Lifshitz, Theory of Elasticity, 2nd ed., Vol. 7 of A Course of Theoretical Physics (Pergamon Press, New York, 1970).

[26] H. C. Hamaker, Physica 4, 1058 (1937).

[27] T. D. Blake and J. M. Haynes, J. Colloid Interface Sci. 30, 421 (1969).

[28] A. Prevost, E. Rolley, and C. Guthmann, Phys. Rev. Lett. 83, 348 (1999).

[29] J. G. Petrov, J. Ralston, M. Schneemilch, and R. A. Hayes, J. Phys. Chem. B 107, 1634 (2003).

[30] R. Fetzer and J. Ralston, J. Phys. Chem. C 114, 12675 (2010).

[31] G. Huber, H. Mantz, R. Spolenak, K. Mecke, K. Jacobs, S. N. Gorb, and E. Arzt, Proc. Natl Acad. Sci. USA 102, 16293 (2005).

[32] Y. Tian, N. Pesika, H. Zeng, K. Rosenberg, B. Zhao, P. McGuiggan, K. Autumn, and J. Israelachvili, Proc. Natl Acad. Sci. USA 103, 19320 (2006).

[33] B. Zhao, N. Pesika, K. Rosenberg, Y. Tian, H. Zeng, P. McGuiggan, K. Autumn, and J. Israelachvili, Langmuir 24, 1517 (2008).

[34] S. A. Wainwright, W. D. Biggs, J. D. Currey, and J. M. Gasoline, Mechanical Design in Organisms (Princeton University Press, Princeton, 1982).

[35] A. M. Peattie, C. Majidi, A. Corder, and R. J. Full, J. R. Soc. Interface 4, 1071 (2007).

[36] R. Ruibal and V. Ernst, J. Morphology 117, 271 (1965).

[37] E. E. Williams and J. A. Peterson, Science 215, 1509 (1982).

[38] G. Huber, S. N. Gorb, R. Spolenak, and E. Arzt, Biol. Lett. 1, 2 (2005).

[39] A. Filippov, V. L. Popov, and S. N. Gorb, J. Theor. Biol. 276, 126 (2011).

[40] T. H. Richards, Energy Methods in Stress Analysis (Ellis Horwood, Chichester, 1977).

[41] E. Marzec, Bioelectroch. Bioener. 46, 29 (1998).

[42] T. Z. Rizvi and M. A. Khan, Int. J. Biol. Macromol. 42, 292 (2008).

[43] W. N. Witzell, Synopsis of Biological Data on the Hawksbill Turtle, Vol. 137 of Fisheries Synopsis (FAO, Rome, 1983). 